\title{
Assessing the Clinical Competence of Health Care Professionals Who Perform Airway Suctioning in Adults
}

\author{
Erin K Miller, Lindsay G Beavers, Brenda Mori, Heather Colquhoun, Tracey JF Colella, and \\ Dina Brooks
}

\author{
Introduction \\ Methods \\ Protocol \\ Identification of Relevant Studies \\ Study Selection \\ Data Extraction \\ Collating, Summarizing, and Reporting the Results \\ Results \\ Literature Search \\ Descriptive Summary \\ Qualitative Directed Content Analysis \\ Discussion \\ Limitations of Current Scoping Review \\ Conclusion
}

\begin{abstract}
Airway suctioning is an important health care intervention that can be associated with serious adverse effects. Given the risks involved with suctioning, it is important to ensure the clinical competence of health care professionals who perform it. A scoping review was conducted to identify the nature and extent of research related to the assessment of airway-suctioning competence for health care professionals working with adults. This included an examination of the assessment context, the type of suctioning and health care professionals being assessed, and the methods used to assess competence. Four scientific electronic databases (MEDLINE, EMBASE, CINAHL, and the Cochrane Library) were searched from inception to March 9, 2018. A gray literature search was also performed. Two reviewers independently screened articles and resources for inclusion, and data were extracted using a form created by the authors. Seventy full text articles and resources were screened for eligibility, with 36 included in the review. Endotracheal suctioning was the most common type, and intensive or critical care units were the primary setting of interest $(28$ of $36,78 \%)$. Competence or a component of competence for nurses, nursing students, nursing assistants, or nurse technicians was specifically addressed in $97 \%$ (35 of 36) of the included articles and resources; 4 of $36(11 \%)$ also included physical therapists, 1 of $36(3 \%)$ included respiratory therapists, and 1 of $36(3 \%)$ was aimed toward all clinicians who perform suctioning. Nine $(25 \%)$ used questionnaire-based assessments, $11(31 \%)$ used checklists, audit forms, or other observational tools, and $16(44 \%)$ used both. Directed content analysis revealed 3 major themes: consistency across overarching evaluation frameworks, inconsistency across detailed components, and inconsistency in the evaluation or reporting of assessment tool measurement properties. Additional gaps in the literature included limited consideration of health care professionals beyond nursing, limited consideration of settings beyond intensive and critical care, a lack of tools to assess nasotracheal and orotracheal suctioning, and limited detail regarding assessment tool
\end{abstract}


development. Key words: suction; trachea; lung; respiratory system; clinical competence; nurses; physical therapists; respiratory therapy; health care providers. [Respir Care 2019;64(7):844-854. (C) 2019 Daedalus Enterprises]

\section{Introduction}

Airway suctioning is a health care intervention that falls within the scope of practice of a number of regulated health care professionals, including physical therapists, respiratory therapists, registered nurses, nurse practitioners, and physicians. ${ }^{1-4}$ This treatment technique involves inserting a suction catheter into an individual's airway to remove retained secretions. For the purpose of this scoping review, the term tracheal suctioning ${ }^{1}$ will be used to refer to suctioning performed via an endotracheal tube, a tracheostomy tube, and the oropharynx or nasopharynx, with or without an artificial airway. Although tracheal suctioning can be a crucial component in maintaining airway patency, it has also been associated with numerous adverse effects, such as tissue trauma, ${ }^{5}$ hypoxia, ${ }^{6}$ and cardiac dysrhythmias. ${ }^{7}$ It is therefore vital to ensure the competence of health care professionals who perform this technique.

Competence in health care can be defined as a health care professional's ability to perform in accordance with a predetermined set of standards. ${ }^{8}$ Competence can be developed in multiple ways, including formal education, inservice training, mentorship opportunities, and clinical experiences. ${ }^{8}$ In addition to technical abilities, competence can also encompass other key elements, such as commu-

Ms Miller is affiliated with the Rehabilitation Sciences Institute, University of Toronto, Toronto, Ontario, Canada. Dr Mori and Ms Beavers are affiliated with the Department of Physical Therapy, University of Toronto, Toronto, Ontario, Canada. Dr Colquhoun is affiliated with the Occupational Science and Occupational Therapy Department, University of Toronto, Toronto, Ontario, Canada. Dr Colella is affiliated with the Cardiovascular Prevention \& Rehabilitation Program, Toronto Rehab, University Health Network; Bloomberg Faculty of Nursing and Rehabilitation Sciences Institute, University of Toronto, Ontario, Canada. Dr Brooks is affiliated with the Rehabilitation Sciences Institute, University of Toronto, Toronto, Ontario, Canada; the School of Rehabilitation Science, McMaster University, Hamilton, Ontario, Canada.

The authors have disclosed a relationship with the Canadian Lung Association Breathing as One Allied Health Fellowship and the Cardiorespiratory Division Scholarship - Canadian Physiotherapy Association, administered by the Physiotherapy Foundation of Canada.

Supplementary material related to this paper is available at http:// www.rcjournal.com.

Correspondence: Erin Miller MScPT MHM PT, Rehabilitation Sciences Institute, University of Toronto, 500 University Avenue, Ontario, Canada, M5G 1V7. E-mail: erin.miller@utoronto.ca.

DOI: $10.4187 /$ respcare. 06772 nication, knowledge, clinical reasoning, values, and reflection on practice. ${ }^{9}$ The provincial regulatory body for physiotherapists in the province of Ontario, Canada, requires physical therapists who perform tracheal suctioning to be evaluated on their relevant knowledge, practical skills, and judgment. ${ }^{10}$ In light of this requirement, knowledge, skills, and judgment will be the key elements used to define clinical competence within this scoping review.

Although a number of surveys have provided insight into the reported suctioning practices of health care professionals, ${ }^{11-15}$ less is known about whether and how competence related to tracheal suctioning is assessed. A search of multiple platforms, including the Joanna Briggs Institute Database of Systematic Reviews and Implementation Reports, the Cochrane Database of Systematic Reviews, Prospero, and PubMed, failed to reveal any systematic or scoping reviews of the literature exploring the assessment of tracheal suctioning competence for health care professionals working with adults. For the purpose of gaining a better understanding of how competence related to tracheal suctioning is being assessed to help promote the safe performance of this high-risk health care activity, we conducted a scoping review. Specifically, we aimed to identify the nature and extent of research related to tracheal suctioning competence assessment for health care professionals working with adults (ie, $\geq 18 \mathrm{y}$ of age). This included an examination of the country of origin, the type of tracheal suctioning being assessed, the setting and context in which it was being assessed, the type of health care professionals being assessed, and the methods used to assess competence. To meet this objective, our review addressed the following questions:

1. What is the nature and extent of assessment being used to evaluate the competence of health care professionals related to the performance of tracheal suctioning (ie, country of origin, setting, context of assessment, type of suctioning, included health care professionals, type of assessment)?

2. What elements are being included in these assessment approaches?

3. What are the similarities and differences across these assessment approaches?

A scoping review methodology was used, given the broad nature of the research objective and questions, the lack of previous comprehensive reviews conducted in this area, and the anticipated heterogeneous nature of the rel- 
evant evidence in terms of the methodological approach, the specific type of tracheal suctioning intervention being assessed, and the type health care professionals included. ${ }^{16}$

\section{Methods}

\section{Protocol}

A protocol for this scoping review was developed based on the 6-stage framework proposed by Arksey and O'Malley, ${ }^{17}$ with the incorporation of modifications suggested by Levac et al. ${ }^{18}$ This protocol is available upon request from the corresponding author.

\section{Identification of Relevant Studies}

Inclusion and Exclusion Criteria. This scoping review included both peer-reviewed and non-peer reviewed publications and resources. To further maximize comprehensiveness, no date limit was set and all study methodologies and resource formats were considered. Articles and resource were included in the review if they met the following inclusion criteria: they used or provided any type of structured approach to assess clinical competence (ie, knowledge, skills and/or judgment) related to tracheal suctioning; and they focused on health care professionals and/or health care professional students.

Exclusion criteria included non-English language resources and resources that described suctioning practices but did not provide a means to assess competence related to tracheal suctioning. Resources pertaining to informal caregivers or family members who perform tracheal suctioning, those dealing specifically with pediatric populations, those dealing exclusively with mouth care or subglottic suctioning devices, and those dealing with ventilator bundle assessments (ie, where a group of related interventions are assessed together) were also excluded because they were outside the direct scope of the primary research objective and questions.

Search Strategy. A comprehensive literature search was conducted by the lead author (EM) and included 4 scientific electronic databases that were searched from inception to March 9, 2018: MEDLINE, EMBASE, Cumulative Index to Nursing and Allied Health Literature (CINAHL), and the Cochrane Library. The initial search strategy was developed in consultation with a librarian, with subsequent review and suggested modifications made to the final search strategy. The search strategy was initially developed and tested in MEDLINE, then refined and translated for use with the other databases. The final search strategy made use of both controlled vocabulary and database-specific search headings (eg, MeSH headings, such as suction/, trachea/ or lung/, and clinical competence), and key word searching (eg, trachea* or airway*, suction*, and competenc* or evaluation* or assess*). A gray literature search was also conducted to locate additional relevant resources that had not been published or archived within the included scientific databases. As well, the reference lists of all included articles and resources were searched to identify additional relevant resources. The final MEDLINE search strategy and the gray literature search strategy are included as supplementary material (Appendix A and B; see the supplementary materials at http://www.rcjournal.com). The detailed search strategies used for the additional electronic databases are available upon request.

\section{Study Selection}

Study screening was performed using the online software Covidence (Covidence, Melbourne, Australia). Two authors (EM, LB) independently reviewed all titles and abstracts. These authors met at the beginning, midpoint, and end of the title and abstract screening process to discuss any challenges or concerns. Consensus was used to resolve any discrepancies, with a third reviewer (DB) available to resolve conflicts as needed. Two authors (EM, LB) then independently reviewed all included full-text articles and other resources to arrive at a final decision for inclusion.

\section{Data Extraction}

A data-extraction form was developed by the authors specifying relevant variables to extract to address the research objective and questions for this scoping review. Two authors (EM, LB) independently extracted data from the first 5 included studies to pilot the data-extraction form and ensure clarity. The finalized data extraction form included article/resource characteristics [type of publication (ie, journal article, clinical manual, academic report, etc.), country of origin, aims/purpose, population, sample size, and setting], type of suctioning intervention, study methodology (if applicable), type of assessment method used, how the assessment method was developed, elements included in the assessment, methods reported or used to assess psychometric properties of any assessment tools, outcomes of interest, and key findings. Data extraction was completed by the lead author using Excel.

\section{Collating, Summarizing, and Reporting the Results}

To address the first and second research questions, a descriptive analysis was conducted, followed by a summary of relevant study characteristics. A map of common elements across the assessment approaches was also produced. To address the third research question (ie, what are the similarities and differences across these assessment 


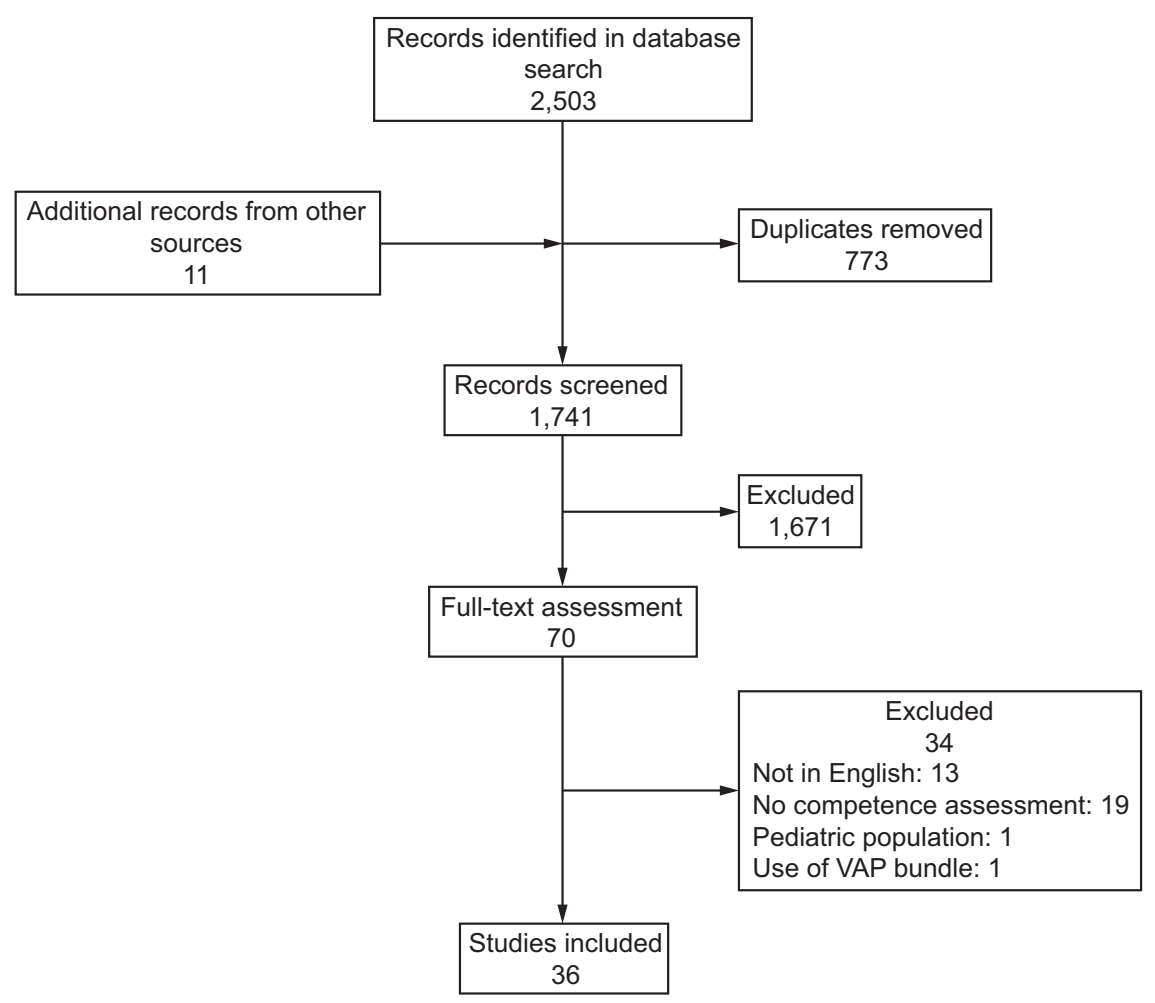

Fig. 1. Flow chart. VAP $=$ ventilator-associated pneumonia.

approaches?), a directed content analysis was conducted by the lead author. A directed content analysis is a qualitative approach used to interpret the meaning of textbased data, using an initial coding scheme developed prior to data analysis. ${ }^{19}$ This was done by examining the extracted data that related to the assessment approaches used, focusing on the similarities and differences across the assessment approaches as an initial coding frame. Using our initial coding frame, the extracted data were then systematically coded to identify themes, highlighting the similarities and differences across the assessment approaches. Extracted data were then revisited to review and refine the identified themes. Findings from the content analysis have been presented by stating and describing the resulting themes. A formal methodological quality assessment for the included studies was not undertaken because scoping reviews are intended to provide a broad view of the existing evidence, regardless of its methodological quality. ${ }^{16}$

\section{Results}

\section{Literature Search}

Comprehensive literature searches of electronic databases yielded 2,503 results, with an additional 11 articles or resources identified through gray literature and reference list searching. Following removal of duplicates and title and abstract screening, 70 records underwent fulltext screening. Thirty-six studies/resources were then included in the review. Results of the comprehensive literature search are presented in Figure 1.

\section{Descriptive Summary}

A descriptive summary of the included articles and resources is provided in Table 1. The included articles and resources ranged in date from 1979 to 2017, with the vast majority ( 33 of $36,92 \%$ ) having been published after the year 2000. Country of origin varied, with multiple articles and resources from each of North America, South America, Asia, Europe, and Australia, and a single study from Africa. India had the single largest contribution to the relevant literature, accounting for $19 \%$ (7 of 36) of the included articles and resources, followed by Brazil ( 5 of 36 , $14 \%$ ), then Iran, the United Kingdom, and the United States, each accounting for $11 \%$ (4 of 36). Endotracheal suctioning was the most common suctioning approach considered, with $67 \%$ (24 of 36 ) of the included studies and resources focusing primarily on the assessment of endotracheal suctioning, using a mix of both open- and closedsystem approaches. Intensive care or critical care units were the primary setting of interest ( 28 of $36,78 \%$ ). Only $2(6 \%)$ of the included studies and resources specifically addressed assessing competence related to oropharyngeal 
Competence Assessment for Airway Suctioning

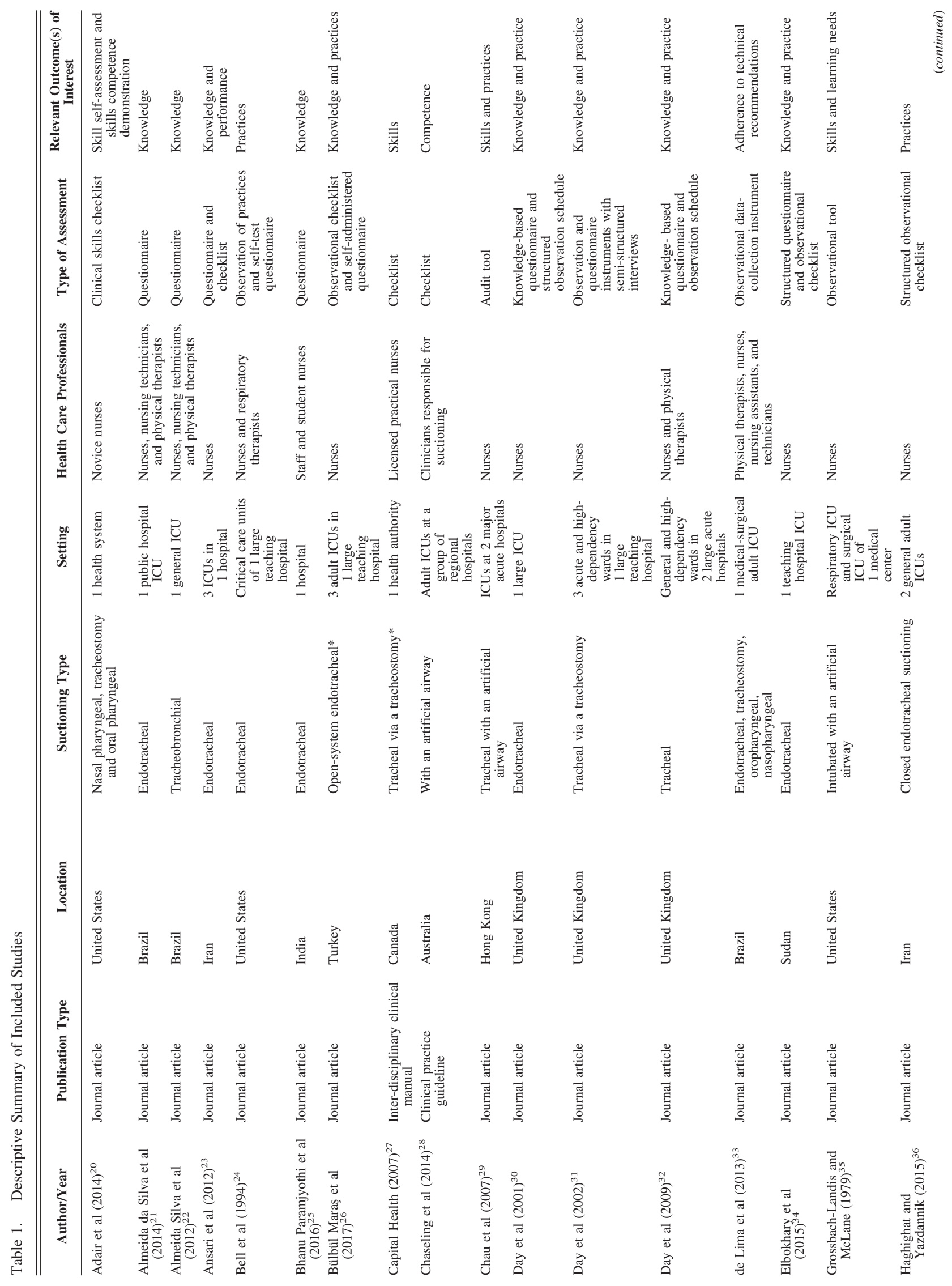




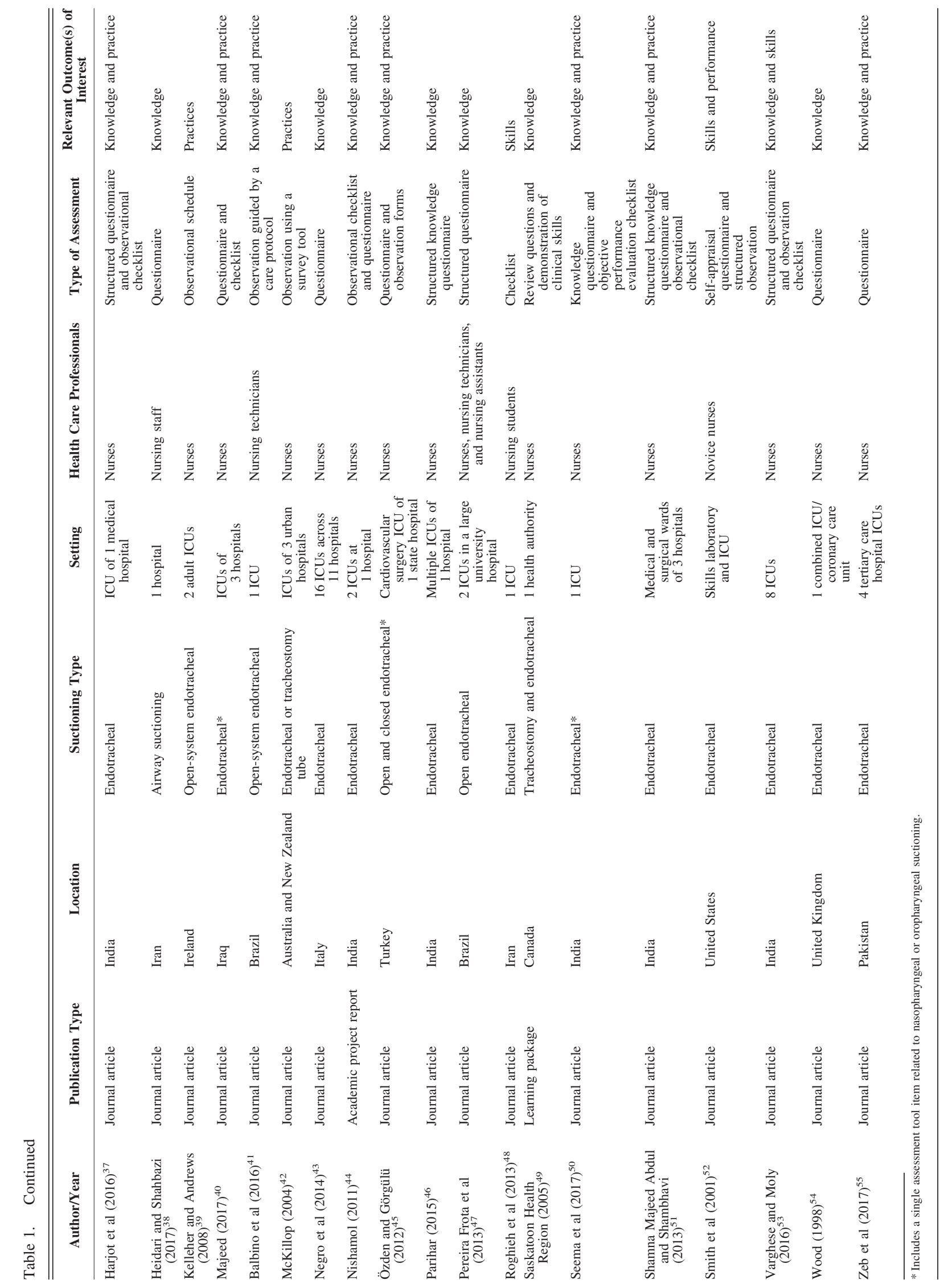


or nasopharyngeal suctioning, although 5 others included a single item related to nasopharyngeal or oropharyngeal suctioning as an adjunct to other types of suctioning. Of the included articles and resources, 97\% (35 of 36) specifically addressed competence assessment or a component of competence assessment related to tracheal suctioning for either nurses, nursing students, nursing assistants, or nurse technicians; while the single remaining resource was aimed more generally at all clinicians who perform suctioning. Four (11\%) of the articles and resources also included physical therapists, whereas respiratory therapists were only included in 1 study (3\%) and physicians were not included in any. In terms of the type of assessment approaches that were used, 25\% (9 of 36) used questionnaire-based assessments (the majority of which contained closed-ended questions and appeared to be self-administered, although this was not always clearly articulated); $31 \%$ (11 of 36) used checklists, audit forms, or other structured observational tools; and the remaining 44\% (16 of 36) used a combination of both questionnaires and observational tools or checklists. A single study also used semistructured interviews to question participants about the reasoning behind their observed practices, in addition to a questionnaire and observational tool. The majority of tools in the included articles and resources were developed or adapted for use as part of a research study, with only $8 \%$ (3 of 36) developed explicitly for use in clinical practice.

\section{Qualitative Directed Content Analysis}

Directed content analysis revealed 3 major themes: 1) Consistency across overarching evaluation frameworks; 2) Inconsistency across components of the tools; and 3) Inconsistency in the evaluation or reporting of assessment tool measurement properties.

\section{Theme 1: Consistency Across Overarching Evaluation} Frameworks. In addition to the consistent use of questionnaire-based and observational/checklist-based approaches, an examination of the overarching assessment frameworks revealed numerous similarities. These similarities included the organizational structure, the nature of the included elements, and the processes used in their development. In terms of organizational structure, assessment tools were most often organized according to the order in which tasks are performed. For example, Haghighat and Yazdannik ${ }^{36}$ grouped items according to practices prior to suctioning, suctioning event practices, and post-suctioning practices, whereas Bülbül Maraş et al ${ }^{25}$ grouped items according to before suctioning, infection-control measures, during suctioning, and post-suctioning. Similarly, Day et $\mathrm{al}^{30,32}$ grouped items according to prior to suctioning, suctioning, and post-suctioning. Even when the included items were not explicitly grouped, a trend toward assess- ment according to the relative sequence of events before, during, and after the performance of suctioning persisted. Balbino et $\mathrm{al}^{41}$ used no formal groupings but organized their assessment with washing hands before the procedure as the first item and recording the characteristics of the secretions in the medical record as the final item. This trend was seen across both checklists/observational tools and questionnaires, with Negro et $\mathrm{al},{ }^{43}$ for example, using a knowledge-based questionnaire to test individuals first on preparing and choosing equipment for suctioning, then focusing later on decision-making during the actual suctioning intervention.

Across the assessment tools, patterns of commonly included assessment elements also emerged. Figure 2 provides a visual representation of these common elements, grouped chronologically. Because hand hygiene and the use of personal protective equipment (PPE) were often considered at multiple points during suctioning, their placement within the figure is intended to convey that they are not confined to a single point in the process. Within this map, the included elements are well aligned with the 3 main components used to define clinical competence within this review (ie, knowledge, skills, and judgment). For example, identifying the need to suction and recognizing contraindications incorporates both knowledge and judgment, and handling the suction catheter is more closely aligned with an individual's skill level.

While numerous (14 of 36, 39\%) articles and resources provided insufficient detail to actually discern how their assessment tools were developed, those articles and resources that did detail the process most often reported developing their tools based on pertinent literature, which often included adapting or making use of other previously developed tools. Kelleher and Andrews ${ }^{39}$ reported collecting data using a structured observational tool that was adapted from a previously established tool created by McKillop. ${ }^{42}$ This same tool used by Kelleher and Andrews ${ }^{39}$ was also then used by Varghese and Moly. ${ }^{53}$ Similarly, Day et $\mathrm{al}^{32}$ used a knowledge-based questionnaire and observation schedule that were previously developed by Day et al. ${ }^{30,31}$ Both adapted tools and new tools that were developed as part of the included articles and resources were frequently informed by existing clinical practice guidelines and systematic reviews, such as those from the American Association for Respiratory $\mathrm{Care}^{56}$ and The Joanna Briggs Institute for Evidence Based Nursing and Midwifery. .57

Theme 2: Inconsistency Across Detailed Assessment Components. Inconsistencies were noted when the finer details of the assessment tools were considered. For example, while patient preparation often included both explaining the procedure to the patient and positioning the patient prior to suctioning, discrepancies existed regarding 


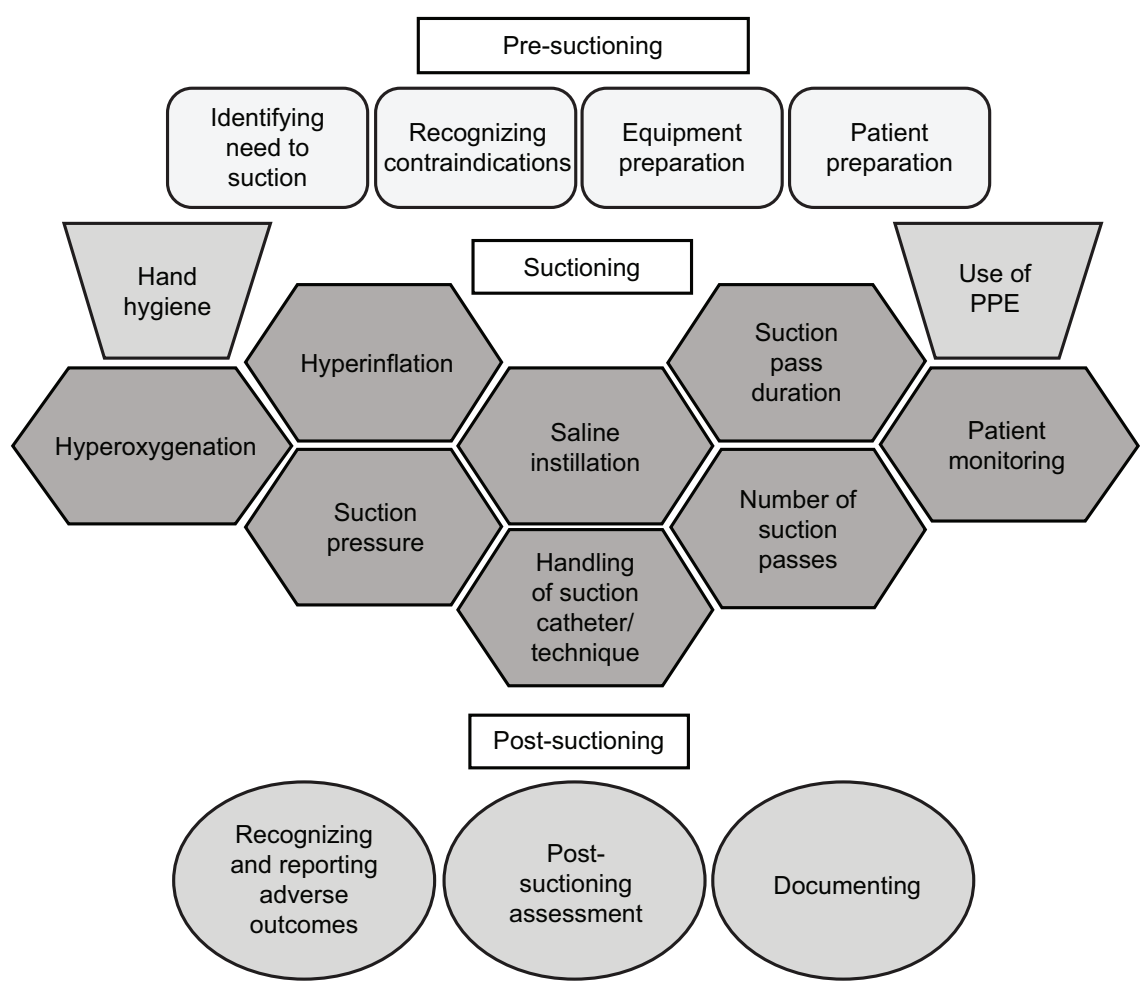

Fig. 2. Map of elements commonly included in the assessment of knowledge, skills and judgment related to suctioning. PPE $=$ personal protective equipment.

the specific position that was recommended. The desired patient position for endotracheal suctioning ranged from Fowler's position ${ }^{34}$ to semi-Fowler's position, ${ }^{45}$ to supine position, ${ }^{50}$ to supine position with the head slightly extended. ${ }^{40}$ Similarly, the recommended target duration for each suction pass, the maximum number of suction passes, and suction pressure all demonstrated variability across the assessment approaches. The maximum target duration for each suction pass included $\leq 10 \mathrm{~s},{ }^{36,45} 10-15 \mathrm{~s},{ }^{29,44}$ and $\leq 15 \mathrm{~s}^{41}$ Meanwhile, the number of suction passes included a maximum of $2,{ }^{29,36}$ a maximum of $3,26,45$ a maximum of $4,{ }^{34}$ and suctioning repeated until the airway is cleared and secretions are removed. ${ }^{50}$ Finally, in terms of suction pressure, Seema et al ${ }^{50}$ suggested $80-100 \mathrm{~mm} \mathrm{Hg}$, Elbokhary et al ${ }^{34}$ Özden and Görgülü̈45 and Capital Health ${ }^{27}$ suggested $80-120 \mathrm{~mm} \mathrm{Hg}$, and Kelleher and Andrews ${ }^{39}$ suggested $80-150 \mathrm{~mm} \mathrm{Hg}$. Numerous assessment tools also contained unique assessment items not found in any other assessment tools, such as locking the suction catheter port, ${ }^{36}$ the use of sterile gauze to clean secretions from the suction catheter, ${ }^{41}$ and chest physiotherapy performed between subsequent suction passes. ${ }^{50}$

Theme 3: Inconsistency in the Evaluation or Reporting of Measurement Properties. Nearly a third (11 of 36, $31 \%$ ) of the included studies and resources did not describe any means used to assess the reliability or validity of the assessment tools. When content validity was explicitly addressed (17 of 36, 47\%), it was most often established based on expert evaluation of the tools. The number of experts involved was generally provided, and on occasion the professional designations and qualifications of the included experts were also given. However, the specific processes used were often described in very limited detail. Evaluation or reporting on the reliability of the tools was also inconsistent. Eight studies (22\%) reported assessing inter-rater reliability, ${ }^{23,26,30-32,41,42,51} 3$ (8\%) reported the use of Cronbach's alpha to assess internal consistency, ${ }^{38,50,51} 3(8 \%)$ reported evaluating test-retest reliability, ${ }^{23,26,40}$ and $2(6 \%)$ reported the use of the split-halves method. ${ }^{36,46}$ One study reported assessing both reliability and validity but provided no further detail. ${ }^{54}$ In addition to the inconsistent evaluation or reporting on reliability and validity, piloting of the tools to address additional issues such as practicality or feasibility was reported for less than half $(16$ of $36,44 \%)$ of the included studies and resources.

\section{Discussion}

This scoping review revealed 36 articles and resources using or providing tools aimed at assessing competence or a component of competence related to the performance of tracheal suctioning for health care professionals working with adults. While the tools originated from a wide variety 
of countries, the similar overarching frameworks likely reflect that many of the tools used common sources of evidence to inform their development and a number of the tools were adapted from one another. Despite these similarities, when individual components were examined in more detail, numerous inconsistencies emerged. These inconsistencies may be rooted in ongoing gaps in the literature pertaining to the optimal performance of tracheal suctioning in both intubated and non-intubated adults. For example, the lack of evidence addressing the appropriate suction pressure, optimal suction pass duration, and use of sterile versus clean technique. ${ }^{58}$ In addition, many of the assessment tools included in this review were intended to be used within specific health care organizations and may have been shaped by organizational or unit-based policies related to tracheal suctioning. In a study conducted across 27 health care facilities in the United States, Sole et al ${ }^{59}$ found that policies related to suctioning vary widely and are not always aligned with current research. This variation across organizational policies may help to further explain some of the discrepancies that are seen between the tools in terms of their specific components.

We also noted inconsistent evaluation and reporting of measurement tool properties. For a testing instrument to be useful, it needs to measure variables of interest in a reliable and valid way. ${ }^{60}$ In the context of tools to assess competence related to tracheal suctioning, certain aspects of validity such as criterion-related validity may not have been feasible to assess given the lack of an acceptedstandard reference. However, even when more feasible aspects of validity were addressed, such as content validity, the lack of detail and transparency often detracted from establishing a strong argument in favor of the tool's use. ${ }^{61}$ In terms of reliability, the use of a second observer to assess inter-rater reliability was the most common technique discussed. This has been reported to be an important consideration, particularly in the context of observational tools where evaluation relies on the judgment of the rater. ${ }^{62}$ Although assessment tools included in this review could have had their measurement properties evaluated in separate corresponding studies, given the comprehensive nature of the search strategy, it is expected that these additional studies would have been found by this review. To promote greater credibility, ${ }^{61}$ it is recommended that future research aimed at assessing the competence of health care professionals related to the performance of tracheal suctioning provide greater detail regarding the processes used for the development of assessment tools and the processes used to establish the measurement properties of the tools for their intended use.

Competence assessment is a critical process for health care professionals, with particular importance being placed on competence assessment for both high-risk and lowvolume health care activities. ${ }^{8}$ Endotracheal suctioning is among the most commonly performed procedures for individuals receiving mechanical ventilation, ${ }^{56}$ but suctioning practice is not limited to individuals on mechanical ventilation or those in intensive care units or other critical care environments. For example, nasotracheal suctioning may be performed across a wide variety of settings in addition to critical care environments, including emergency departments, in-patient acute care units, extended care environments, skilled nursing facilities, out-patient or ambulatory care settings, and during the provision of home care. ${ }^{63}$ In these environments, competence assessment may be of particular importance if suctioning constitutes a lower-volume activity, performed less frequently in practice.

Tools identified in this review rarely aimed to address tracheal suctioning competence for health care professionals beyond nursing and nursing-related professions. They also did not reflect the wide variety of settings in which tracheal suctioning can be performed, and they rarely considered nasopharyngeal/nasotracheal and oropharyngeal/orotracheal suctioning. Future research should consider the assessment of competence related to tracheal suctioning for health care professionals beyond nursing and nursing-related professions. There is also a need to focus on assessment approaches that can be applied across different health care settings and that can be used to evaluate a broader variety of suctioning approaches. Researchers, clinicians, and educators will need to consider whether current tools can be used or adapted for use within their individual contexts, and whether the creation of new tools is required to meet their needs.

\section{Limitations}

This scoping review used a transparent, systematic, and reproducible process, guided by a pre-established framework ${ }^{17,18}$ and a protocol developed by the authors. Although a comprehensive search was conducted, assessment tools which had not been published or shared through an accessible electronic source may have been missed. Additional articles and resources may also have been missed due to the exclusion of non-English-language documents. In the interest of comprehensiveness, we chose to set no date limit for our results, nor did we limit the included studies/resources based on country of origin. However, given that numerous included studies were published prior to more recent clinical practice guidelines for suctioning, this could have contributed to some of the variability in our findings. Similarly, heterogeneity of study origin may have contributed additional variability, given the potential for regional variation in terms of recommended or acceptable practices. In addition, although the gray literature search and the directed content analysis were performed 
by a single author, the processes followed and the resulting findings were reviewed in detail by all team members. Finally, the sixth step in conducting a scoping review, as outlined by both Arksey and O'Malley ${ }^{17}$ and Levac et al, ${ }^{18}$ (ie, consulting with experts) was not undertaken within the current review. We acknowledge the inherent value in this step, and expert consultation regarding the assessment of competence related to tracheal suctioning is being incorporated into a future study.

\section{Conclusion}

A variety of tools to assess competence or a component of competence related to tracheal suctioning for health care professionals working with adults were identified within this scoping review. Although these tools shared consistent overarching frameworks in terms of their organizational structure, the nature of the included elements, and the processes used in their development, inconsistencies were evident when individual assessment items were examined in greater detail. The vast majority of the tools were designed to be used with nurses or nursing-related professions, and their appropriateness for use wither other health care professionals is unclear. Further gaps in the literature included a lack of tools to assess tracheal suctioning performed outside of intensive care or other high acuity hospital-based units, a lack of tools to assess nasotracheal and orotracheal suctioning, and limited detail regarding tool development and the evaluation of measurement properties. Future research is recommended to address these limitations.

\section{ACKNOWLEDGMENTS}

We thank Erica Lenton for her assistance in developing and refining the search strategy that was used for this scoping review.

\section{REFERENCES}

1. Government of Ontario. Physiotherapy Act, 1991, S.O. 1991, c. 37. 2011. Available at: https://www.ontario.ca/laws/statute/91p37. Accessed May 15, 2018.

2. Government of Ontario. Respiratory Therapy Act, 1991, S.O. 1991, c. 39. 2009. Available at: https://www.ontario.ca/laws/statute/91r39. Accessed May 15, 2018.

3. Government of Ontario. Nursing Act, 1991, S.O. 1991, c. 32. 2017. Available at: https://www.ontario.ca/laws/statute/91n32. Accessed May 15, 2018.

4. Government of Ontario. Medicine Act, 1991, S.O. 1991, c. 30.2017. Available at: https://www.ontario.ca/laws/statute/91m30. Accessed May 15, 2018.

5. Runton N. Suctioning artificial airways in children: appropriate technique. Pediatr Nurs 1992;18(2):115-118.

6. Abbasinia M, Irajpour A, Babaii A, Shamali M, Vahdatnezhad J. Comparison the effects of shallow and deep endotracheal tube suctioning on respiratory rate, arterial blood oxygen saturation and number of suctioning in patients hospitalized in the intensive care unit: a randomized controlled trial. J Caring Sci 2014;3(3):157-164.
7. Johnson KL, Kearney PA, Johnson SB, Niblett JB, MacMillan NL, McClain RE. Closed versus open endotracheal suctioning: costs and physiologic consequences. Crit Care Med 1994;22(4):658-666.

8. Kak N, Burkhalter B, Cooper M. Measuring the competence of healthcare providers. Bethesda, MD: Operations Research Issue Paper; 2001.

9. Epstein RM, Hundert EM. Defining and assessing professional competence. JAMA 2002;287(2):226-235.

10. College of Physiotherapists of Ontario. Controlled acts and restricted activities standard: 2016. Available at: https://www.collegept.org/ rules-and-resources/controlled-acts-and-restricted-activities. Accessed Nov 9, 2018.

11. Brooks D, Solway S, Graham I, Downes L, Carter M. A survey of suctioning practices among physical therapists, respiratory therapists and nurses. Can Respir J 1999;6(6):513-520.

12. Leddy R, Wilkinson JM. Endotracheal suctioning practices of nurses and respiratory therapists: how well do they align with clinical practice guidelines? Can J Respir Ther 2015;51(3):60-64.

13. Cross J. A survey of physiotherapists' current practice with closed suction systems in adult patients. Adv Physiother 2001;3(2):67-75.

14. Sole ML, Bennett M. Comparison of airway management practices between registered nurses and respiratory care practitioners. Am J Crit Care 2014;23:191-200.

15. Tan CS, Harrold M, Hill K. Approaches and adjuncts used by physiotherapists when suctioning adult patients who are intubated and ventilated in intensive care units in Australia and New Zealand: a cross-sectional survey. Aust Crit Care 2017;30(6):307-313.

16. Peters MD, Godfrey CM, Khalil H, McInerney P, Parker D, Baldini Soares C. Guidance for conducting systematic scoping reviews. Int J Evid Based Healthc 2015;13(3):141-146.

17. Arksey H, O’Malley L. Scoping studies: towards a methodological framework. Int J Social Res Methodol 2005;8(1):19-32.

18. Levac D, Colquhoun H, O'Brien KK. Scoping studies: advancing the methodology. Implement Sci 2010;5:69.

19. Hsieh H-F, Shannon SE. Three approaches to qualitative content analysis. Qual Health Res 2005;15(9):1277-1288.

20. Adair J, Hughes L, Davis S, Wolcott-Breci M. Comparing new BSN $\mathrm{RN}$ self skills assessment to actual skills demonstration. J Prof Nurs 2014;30(2):180-184.

21. Almeida da Silva L, Souza Pacheco JA, Silva LL, Fassini Mantelli F, Silveira SE, Parreira Oliveira L. Endotracheal aspiration: intensivist professional knowledge. J Nurs UFPE 2014;8(8):2676-2685.

22. Almeida Silva L, Sampaio Teles LC, do Carmo Cruz Robazzi ML, Elias Silveira S, Souza Terra F. Knowledge of tracheobronchial aspiration of health professionals from the intensive care unit. J Nurs UFPE 2012;6(11):2625-2632.

23. Ansari A, Masoudi Alavi N, Adib-Hajbagheri M, Afazel M. The gap between knowledge and practice in standard endo-tracheal suctioning of ICU nurses, Shahid Beheshti Hospital. Iran J Crit Care Nurs 2012;5(2):71-76.

24. Bell S, Taggart J, Karl J, Lind M, Peterman P, Stone K. Implementing a research-based protocol: an interactive approach. AACN Clin Issues Criti Care Nurs 1994;5(2):147-151.

25. Bhanu Paramjyothi B, Daniel LE, Indira S. A study to assess the knowledge regarding ET suctioning among staff nurses and student nurses in NMCH, Nellore. Int J Appl Res 2016;2(6):150-152.

26. Bülbül Maraş G, Kocaçal Güler E, Eşer İ, Köse Ş. Knowledge and practice of intensive care nurses for endotracheal suctioning in a teaching hospital in western Turkey. Intensive Crit Care Nurs 2017; 39:45-54.

27. Capital Health. Tracheostomy, care and management of patients with tracheostomy. Interdisciplinary Clinical Manual Policy \& Procedure: May, 2007. Available at: http://policy.nshealth.ca/Site_Published/ DHA9/document_render.aspx?documentRender.IdType $=6 \&$ document 


\section{Competence Assessment for Airway Suctioning}

Render.GenericField $=\&$ documentRender.Id=48038. Accessed March 20, 2018.

28. Chaseling W, Bayliss S-L, Rose K, Armstrong L, Boyle M, Caldwel $\mathrm{J}$ et al. Suctioning an Adult ICU patient with an artificial airway. Agency for Clinical Innovation NSW Government Version 2 Chatswood, NSW, Australia. 2014;33.

29. Chau J, Thompson DR, Chan D, Chung L, Wai-Lin A, Tam S, et al. An evaluation of the implementation of a best practice guideline on tracheal suctioning in intensive care units. Int J Evid Based Healthc 2007;5(3):354-359.

30. Day T, Wainwright S, Wilson-Barnett J. An evaluation of a teaching intervention to improve the practice of endotracheal suctioning in intensive care units. J Clin Nurs 2001;10:682-696.

31. Day T, Farnell S, Haynes S, Wainwright S, Wilson-Barnett J. Tracheal suctioning: an exploration of nurses' knowledge and competence in acute and high dependency ward areas. J Adv Nurs 2002; 39(1):35-45.

32. Day T, Iles N, Griffiths P. Effect of performance feedback on tracheal suctioning knowledge and skills: randomized controlled trial. J Adv Nurs 2009;65(7):1423-1431.

33. de Lima ED, Fleck CS, Borges JJ, Condessa RL, Vieira SR. Effects of educational intervention on adherence to the technical recommendations for tracheobronchial aspiration in patients admitted to an intensive care unit. Rev Bras Ter Intensiva 2013;25(2):115-122.

34. Elbokhary R, Osama A, AL-kharder M. Knowledge and practice of ICU nurses regarding endotracheal suctioning for mechanically ventilated patients in Khartoum Teaching Hospital. Am J Cli Neurol Neurosurg 2015;1(2):92-98.

35. Grossbach-Landis I, McLane AM. Tracheal suctioning: a tool for evaluation and learning needs assessment. Nurs Res 1979;28(4):237242.

36. Haghighat S, Yazdannik A. The practice of intensive care nurses using the closed suctioning system: an observational study. Iranian J Nurs Midwifery Res 2015;20(5):619-625.

37. Harjot K, Hemant Kumar S, Krishan GK. Effectiveness of teaching intervention on knowledge and practices regarding endotracheal tube suctioning among staff nurses. Int J Nurs Educ 2016;8(2):8-11.

38. Heidari M, Shahbazi S. Nurses' awareness about principles of airway suctioning. J Clin Diagn Res 2017;11(8):LC17-LC19.

39. Kelleher S, Andrews T. An observational study on the open-system endotracheal suctioning practices of critical care nurses. J Clin Nurs 2008;17(3):360-369.

40. Majeed HM. Assessment of knowledge and practices of intensive care unit nurses about endotracheal suctioning for adult patients in Baghdad teaching hospitals, Iraq. Int J Res Med Sci 2017;5:4.

41. Balbino CM, Ribeiro Braz M, de Castro Medeiros J, Simões Rodrigues LM, Silvino ZR. Evaluation of aspiration technique on the patient with mechanical ventilation performed by nursing. J Nurs UFPE 2016;10:4797-4803

42. McKillop A. Evaluation of the implementation of a best practice information sheet: tracheal suctioning of adults with an artificial airway. Int J Evid Based Healthc 2004;2(9):293-308.

43. Negro A, Ranzani R, Villa M, Manara D. Survey of Italian intensive care unit nurses' knowledge about endotracheal suctioning guidelines. Intensive Crit Care Nurs 2014;30(6):339-345.

44. Nishamol Y. A study to assess the knowledge and practice of endotracheal suctioning among neuronurses SCTISMT. Project Report: Nov 2011. Available at: http://dspace.sctimst.ac.in/jspui/bitstream/ 123456789/1609/1/483.pdf. Accessed March 17, 2018.

45. Özden D, Görgülü RS. Development of standard practice guidelines for open and closed system suctioning. J Clin Nurs 2012;21(9/10): 1327-1338.
46. Parihar R. Effectiveness of planned teaching programme on the knowledge of endotracheal suctioning among staff nurses working in ICU. Baba Farid University Nurs J 2015;8(1):63-67.

47. Pereira Frota O, Dias Rolan Loureiro M, Menis Ferreira A. Knowledge about endotracheal suctioning on the part of intensive care nursing professionals: a descriptive study. Online Braz J Nurs 2013; 12(3):546-554.

48. Roghieh N, Fateme H, Hamid S, Hamid H. The effect of formative evaluation using "direct observation of procedural skills" (DOPS) method on the extent of learning practical skills among nursing students in the ICU. Iran J Nursing Midwifery Res 2013;16(4):290293.

49. Saskatoon Health Region. Suctioning artificial airways in adults: tracheostomies and endotracheal tubes. RN and LPN Learning Package: Jun 2005. Available at: https://www.saskatoonhealthregion.ca/ joinourteam/nursing/Documents/Orientation\%20Information/ suctioning_artificial_airways_-_adults.pdf. Accessed Mar 20, 2018.

50. Seema S, Pity K, Kiran B. Effectiveness of suction protocol on nurse's and patient's outcome in ICU. Asian J Nurs Educ Res 2017; 7(4):589-595.

51. Shamna Majeed Abdul M, Shambhavi. Effectiveness of planned teaching programme on knowledge and practice of endotracheal suctioning among staff nurses in selected hospitals of Mangalore. Asian J Nurs Educ Res 2013;3(4):243-247.

52. Smith AM, Ortiguera SA, Laskowski ER, Hartman AD, Mullenbach DM, Gaines KA, et al. A preliminary analysis of psychophysiological variables and nursing performance in situations of increasing criticality. Mayo Clin Proc 2001;76(3):275-284.

53. Varghese S, Moly K. Exploratory study on the knowledge and skill of critical care nurses on endotracheal suctioning. J Nat Accred Board Hosp Healthc Providers 2016;3(1):13-19.

54. Wood CJ. Can nurses safely assess the need for endotracheal suction in short-term ventilated patients, instead of using routine techniques? Intensive Crit Care Nurs 1998;14(4):170-178.

55. Zeb A, Shams-ul-Haq, Ali F, Hussain N, Haidar Ali Shah S, Faisal S. Knowledge and practice of ICU nurses regarding endotracheal suctioning in tertiary care hospitals, Peshawar. JOJ Nurse Health Care 2017;2(4):555-595.

56. American Association for Respiratory Care. AARC clinical practice guidelines. Endotracheal suctioning of mechanically ventilated patients with artificial airways 2010. Respir Care 2010;55(6):758-764.

57. Thompson L. Suctioning adults with an artificial airway: a systematic review. The Joanna Briggs Institute for Evidence Based Nursing and Midwifery. Adelaide, S. Australia, Australia: 2000;9.

58. Overend TJ, Anderson CM, Brooks D, Cicutto L, Keim M, McAuslan D, Nonoyama M. Updating the evidence-base for suctioning adult patients: a systematic review. Can Respir J 2009;16(3):e6-e17.

59. Sole ML, Byers JF, Ludy JE, Zhang Y, Banta CM, Brummel K. A multisite survey of suctioning techniques and airway management practices. Am J Crit Care 2003;12(3):220-230.

60. Karras JD. Statistical methodology. II. Reliability and validity assessment in study design, part A. Acad Emerg Med 1997;4(1):64-71.

61. Sullivan GM. A primer on the validity of assessment instruments. J Grad Med Educ 2011;3(2):119-120.

62. Kimberlin CL, Winterstein A. Validity and reliability of measurement instruments used in research. Am J Health-Syst Pharm 2009; 65:2276-2284.

63. American Association for Respiratory Care. AARC clinical practice guideline. Nasotracheal suctioning — 2004 revision \& update. Respir Care 2004;49(9):758-764. 